\title{
The Congruency of Neuropsychological and F18-FDG Brain PET/CT Diagnostics of Alzheimer's Disease (AD) In Routine Clinical Practice: Insights From a Mixed Neurological Patient Cohort
}

Sascha Hansen ( $\sim$ sascha.hansen@klinikum-bayreuth.de)

Klinikum Bayreuth $\mathrm{GmbH}$

Jana Keune

Klinikum Bayreuth $\mathrm{GmbH}$

Kim Küfner

University of Bamberg

Regina Meister

Klinikum Bayreuth GmbH

Juliane Habich

Klinikum Bayreuth GmbH

Julia Koska

Klinikum Bayreuth GmbH

Stefan Förster

Klinikum Bayreuth GmbH

Patrick Oschmann

Klinikum Bayreuth GmbH

Philipp M. Keune

Klinikum Bayreuth $\mathrm{GmbH}$

\section{Research Article}

Keywords: Neuropsychological diagnostics, Cognition, Alzheimer 's Disease, FDG-PET/CT.

Posted Date: June 3rd, 2021

DOl: https://doi.org/10.21203/rs.3.rs-561849/v1

License: (c) (i) This work is licensed under a Creative Commons Attribution 4.0 International License. Read Full License

Version of Record: A version of this preprint was published at BMC Neurology on March 9th, 2022. See the published version at https://doi.org/10.1186/s12883-022-02614-4. 


\section{Abstract}

Background:

Clinical diagnostics of Alzheimer's Disease (AD) require a multimodal approach. Neuropsychological assessments are commonly implemented to obtain information about the degree of cognitive impairment, while cerebrospinal fluid markers and imaging data provide etiological information. In routine clinical practice, neuropsychologists often have to rely on relatively limited anamnestic information, as well as cognitive test results and are required to infer whether patients actually suffer from $A D$, prior to the availability of exhaustive etiological information. To date, it remains to be explored how congruent the results of such a phenomenological approach may be with results from neuroimaging techniques such as FDG$\mathrm{PET} / \mathrm{CT}$ examinations. The latter are known to yield highly accurate diagnostic information.

Methods:

A mixed sample of $\mathrm{N}=127$ hospitalized neurological patients suspected of displaying a syndrome of dementia underwent routine differential diagnostics including an extensive neuropsychological and an FDGPET/CT examination. The neuropsychological examination included an interview in which anamnestic information was obtained, as well as the administration of the standardized CERAD cognitive test battery. Two separate decisional approaches were considered: First, routine diagnostic results were obtained, as reflected by the final clinical decision of the examining neuropsychologist $\left(A D_{\text {Clinical }} v s\right.$. non- $\left.A D_{\text {Clinical }}\right)$ for the routine clinical report. Secondly, a logistic regression model was implemented, that relied on data from the CERAD test profiles alone. Based on the logistic regression, the CERAD test subscales that best predicted the presence of $A D$ according to the FDG-PET/CT results were identified and a nominal categorization was obtained $\left(A D_{\text {Test }} v s\right.$. non- $\left.A D_{\text {Test }}\right)$. Results from both decisional approaches were matched against the FDG$P E T / C T$ results $\left(A D_{P E T}\right.$ vs. non- $\left.A D_{P E T}\right)$ in cross-tables and estimates of accuracy, sensitivity and specificity were derived.

Results:

Based on the FDG-PET/CT examination, $\mathrm{N}=33 / 127$ (26\%) of the patients were diagnosed as $A D_{P E T}$ patients. When matched against these results, the clinical decision approach of the neuropsychological examination $\left(A D_{\text {Clinical }}\right.$ vs. non- $\left.A D_{\text {Clinical }}\right)$ yielded a good accuracy (84.2\%), involving moderate sensitivity $(75.8 \%)$ and excellent specificity (92.6\%). The decisional approach that relied on the neuropsychological test data alone $\left(A D_{\text {Test }}\right.$ vs. non- $\left.A D_{\text {Test }}\right)$ involved a lower estimate of accuracy $(74.8 \%)$, that was attributable to considerably decreased sensitivity $(56.3 \%)$ while specificity was comparable $(93.3 \%)$ to the clinical decision model.

Conclusions:

These results indicate that it is feasible to identify $A D$ in context of a comprehensive routine neuropsychological examination in a mixed sample of neurological patients, relative to an FDG-PET/CT classification. However, decisions based on cognitive test results alone appear limited in this respect. It may be assumed that anamnestic information in combination with the clinical impression obtained by the neuropsychological examiner play a crucial role in the identification of AD patients in routine clinical practice. 


\section{Background}

Alzheimer's disease (AD) is considered to be the most common type of dementia, with prevalence rates drastically increasing from below $1 \%$ for patients aged 65 to 74 to above $22 \%$ for patients aged 85 and older [1]. Cognitive decline, beginning with short-term memory impairment, is the leading and most debilitating symptom of $A D$ with an onset early in the disease [2]. Valid tools to assess cognitive deficits are neuropsychological test batteries such as the Consortium to Establish a Registry on Alzheimer's Disease Neuropsychological Assessment Battery (CERAD-NAB) [3]. However, since cognitive deficits constitute a common symptom in many neurological diseases, the specificity of generalized cognitive decline when differentiating between different forms of dementia is inevitably negligible. In order to successfully differentiate between different forms of dementia, clinical symptoms have to be assessed in closer detail. This is feasible via neuropsychological test batteries such as the CERAD-NAB, that assess cognitive deficits in several domains such as attention, memory, executive function, language, motor praxis and visuo-spatial perception. The validity of this approach has been evaluated positively in numerous studies $[4,5,6]$, although some limitations have also been reported [7]. Neuropsychologists can usually rely on additional clinical information regarding anamnesis, behavioral monitoring during the administration of the neuropsychological tests and information from family members or next-of-kin. These may also serve as an important source of information when determining the most likely diagnosis, albeit they may not always be systematically obtained or reported during routine clinical practice $[8,9]$.

Neuroimaging techniques, on the other hand, have become a complementary approach to differential diagnostics with increasing accuracy. Concerning the etiological differentiation between $A D$ and nondemented controls, FDG-PET/CT may play an important role in establishing a diagnosis, as its' predictive value has been determined as excellent in this regard [10]. Moreover, FDG-PET/CT also has the ability to differentiate between different types of dementia with a high accuracy [11,12]. FDG-PET/CT represents a minimally invasive imaging procedure that measures the regional distribution of cerebral metabolic glucose. Specific patterns of regional glucose reduction allow inferences on the etiology of the underlying disease [13].

In routine clinical practice, it remains to be determined in detail which aspects of the neuropsychological assessment contribute to the accuracy of the differential diagnostic decision. In recent studies, sensitivity and specificity of neuropsychological diagnostics reached reasonably high values [5,6], but these results were based on information from both, neuropsychological assessments as well as additional information gained during the clinical process. On the other hand, research suggests that specific deficit profiles exist that might allow a differentiation based on test results alone $[4,14]$. For instance, in $A D$, short-term memory decline is reported to be the leading cognitive symptom at least in the early stages of the disease [2].

Based on these considerations, the purpose of the current study was to examine the congruency of neuropsychological diagnostics and FDG-PET/CT diagnostics, in terms of differentiating between AD versus non-AD patients in a mixed neurological sample. With regards to the neuropsychological diagnostics, two decisional approaches were implemented: The first approach was a clinical decision approach $\left(A D_{\text {Clinical }} v s\right.$.

non- $\left.A D_{\text {Clinical }}\right)$. This approach considered information from the standardized neuropsychological assessment as well as all additional unstandardized anamnestic and behavioral information gleaned during the neuropsychological examination. In essence, this approach reflects the final decision of the clinical 
neuropsychologist as it occurred in routine clinical practice. The second approach relied on a logistic regression model, based on the neuropsychological test results alone $\left(A D_{\text {Test }}\right.$ vs. non- $\left.A D_{\text {Test }}\right)$. Results of both decisional approaches were matched against the FDG-PET/CT neuroimaging diagnosis. It was assumed that both decisional approaches would yield sufficient predictive values in terms of the differential diagnosis (AD vs. non-AD), relative to the FDG-PET/CT diagnosis. It was further expected that results of the logistic regression model would be in line with the extant literature on characteristic cognitive deficit profiles in AD. Nevertheless, it was also assumed that results of the regression model would be inferior to the more comprehensive clinical approach in differentiating between $A D$ and non-AD patients. The latter hypothesis implies that the overall clinical impression of the neuropsychologist, that incorporates anamnestic information, test results and behavioral monitoring during test administration, is of critical relevance for the differential diagnostic decision.

\section{Methods}

The CERAD-NAB was administered to 127 inpatients at the Department of Neurology, Klinikum Bayreuth $\mathrm{GmbH}$, Germany, during the routine clinical process. Testing was conducted by highly practiced psychologists specialized in the field of neuropsychology. Neuropsychological assessments also included an unstandardized interview to obtain basic anamnestic information. Additionally, patients underwent an extensive neurological assessment including neuroimaging diagnosis via FDG-PET/CT. Inclusion criteria involved suspected cognitive decline observed upon admission to the hospital that resulted in a referral to the department of neuropsychology for further testing, as it was assumed to be compatible with the presence of a syndrome of dementia according to ICD-10 [15], i.e. impaired memory and other cognitive functions, unclouded consciousness, as well as a deterioration in motivation, social behavior or emotional control. The etiology of cognitive decline had to be unclear at the time of hospitalization. Patients' age had to fall in the age range covered by CERAD-norms (49-92 years). Patients were not eligible for inclusion if they had severe visual or hearing impairments that interfered with cognitive testing, or if they had less than eight years of formal education. They were also not eligible for study entry if they lacked the capacity to consent to participate due to cognitive decline. A flow diagram detailing patient recruitment can be reviewed in Appendix 1. All participants provided written informed consent prior to study entry. The study was approved by the ethics committee of the University of Bamberg, Germany (reference number 2019-02/10). Demographic and clinical information of the sample is displayed in Table 1.

Table 1 Demographic and clinical information of the study sample 


\begin{tabular}{|c|c|c|c|c|c|c|c|c|}
\hline Groups & $A D$ & $\begin{array}{l}\text { non- } \\
A D\end{array}$ & & & $\begin{array}{l}\text { non-AD } \\
\text { subgroups }\end{array}$ & & & $\begin{array}{l}p- \\
\text { value }\end{array}$ \\
\hline & & & PD/PDD & LBD & VD & Unconspicuous & Other & $\begin{array}{l}A D \\
\text { vs. } \\
\text { non- } \\
A D\end{array}$ \\
\hline $\begin{array}{l}\text { Participants } \\
\text { (N) }\end{array}$ & 33 & 94 & 27 & 16 & 14 & 15 & 22 & \\
\hline $\begin{array}{l}\text { Sex (N: } \\
\text { male/female) }\end{array}$ & $18 / 15$ & $53 / 41$ & $16 / 11$ & $13 / 3$ & $6 / 8$ & $8 / 7$ & $10 / 12$ & .856 \\
\hline $\begin{array}{l}\text { Age (years: } \\
\text { M, SD) }\end{array}$ & $\begin{array}{l}\text { 76.70, } \\
8.29\end{array}$ & $\begin{array}{l}74.33 \\
8.36\end{array}$ & $\begin{array}{l}73.81 \\
7.95\end{array}$ & $\begin{array}{l}77.94 \\
4.91\end{array}$ & $\begin{array}{l}77.93 \\
7.09\end{array}$ & $77.40,6.07$ & $\begin{array}{l}67.95 \\
9.48\end{array}$ & .163 \\
\hline $\begin{array}{l}\text { Education } \\
\text { (years: M, } \\
\text { SD) }\end{array}$ & $\begin{array}{l}11.09 \\
1.91\end{array}$ & $\begin{array}{l}12.53 \\
2.98\end{array}$ & $\begin{array}{l}11.89 \\
2.72\end{array}$ & $\begin{array}{l}14.44 \\
3.35\end{array}$ & $\begin{array}{l}11.14 \\
2.18\end{array}$ & $12.80,2.93$ & $\begin{array}{l}12.64 \\
2.95\end{array}$ & $.011^{*}$ \\
\hline $\begin{array}{l}\text { MMSE } \\
\text { (score: M, } \\
\text { SD) }\end{array}$ & $\begin{array}{l}23.76 \\
4.01\end{array}$ & $\begin{array}{l}25.30 \\
3.66\end{array}$ & $\begin{array}{l}26.00 \\
2.71\end{array}$ & $\begin{array}{l}22.13 \\
4.51\end{array}$ & $\begin{array}{l}25.57 \\
3.69\end{array}$ & $27.06,1.03$ & $\begin{array}{l}25.43 \\
3.93\end{array}$ & $.040 *$ \\
\hline
\end{tabular}

Note. Classification of $A D$ versus non-AD and non-AD subgroups based on FDG-PET/CT results. $A D=A l z h e i m e r$ Disease; $L B D=L e w y$ Body Dementia; MMSE=Mini Mental Status Examination; $P D=P a r k i n s o n$ Disease; PDD=Parkinson Disease Dementia; $V D=$ Vascular Dementia; *significant at $p$-value $<.05$, two-tailed, based on one-way ANOVA model. In the non-AD subgroups, the subgroup "other" involved reports of functional anomalies suspected to be of cancerous or post-operative etiology $(n=7)$, progressive supranuclear palsy $(n=4)$, normal pressure hydrocephalus $(n=3)$, multiple system atrophy type $C(n=3)$, frontotemporal dementia $(n=2)$ and unspecified etiology $(n=3)$.

\section{Neuropsychological Assessment}

The German version of the CERAD-NAB [3] (CERAD-Plus [6]) was implemented for the neuropsychological examination. The CERAD-Plus consists of the nine basic tests included in the CERAD-NAB, as well as three additional tests assessing executive functions. The 12 tests included in the CERAD-Plus assess the following cognitive domains: verbal and nonverbal memory, verbal fluency, language, praxis, orientation, cognitive flexibility, psychomotor speed and visual scanning. For a detailed description of all subtests please see [16].

\section{Brain PET/CT Imaging Procedure}

All patients underwent 18F-FDG brain PET/CT scans in a resting state. A mean of 181 MBq F18-FDG was administered intravenously under standardized conditions in a quiet, dimly lit room with patients' eyes open. The procedure took place in a neuroimaging center experienced with PET (Department for Nuclear Medicine, Klinikum Bayreuth $\mathrm{GmbH}$, Germany). A 10-minute 3D-listmode PET emission scan was acquired at mean 40 minutes post injection in one bed position using a state-of-the-art Siemens Biograph mCT PET/CT scanner with extended field of view (Siemens Healthineers, Erlangen, GER). During the scanning procedure, patients' heads were immobilized using a head holder. Attenuation correction was performed using low-dose CT (100 
mAs, $120 \mathrm{kV}$, Collimation 40x0,6mm) before the PET emission scan. Following corrections for scatter, dead time, and random coincidences, PET images were reconstructed by using 3-dimensional filtered back projection, Gaussian Filter, FWHM 3.5mm. Finally, reconstructed PET data was post-processed on a syngo Via workstation (VB10) using the MI Neurology application. Images were clinically analyzed by a reader experienced in neuroimaging. Analyses took place slice-wise, surface projections-wise, including comparisons with an age-matched, scanner-matched healthy control group, as well as PET/MR fusion-image wise (after coregistration of PET and MRI data and fusion of PET and T2w FLAIR brain MR images).

\section{Statistical Analysis}

In order to obtain information about the congruency of results of the neuropsychological examination and the FDG-PET/CT diagnostics, two approaches were implemented. The first one considered a decision model, in which the clinical neuropsychological diagnoses were based on the comprehensive final decision of the examining neuropsychologist. This model hence included the clinical decision whether AD was present or not $\left(A D_{\text {Clinical }}\right.$ vs. non- $\left.A D_{\text {Clinical }}\right)$, based on anamnestic information obtained during the examination, behavioral observation during testing, as well as the evaluation of the test results themselves. Dichotomous results of this decision model were matched against the dichotomous FDG-PET/CT diagnostic results (AD ${ }_{\text {PET }}$ Vs. non-

$\left.A D_{P E T}\right)$ by means of a cross-tabulation to obtain estimates of sensitivity, specificity and accuracy. To account for a potential asymmetric distribution in the classification of cases, a balanced accuracy statistic was computed as the mean of sensitivity and specificity estimates.

In the second approach, neuropsychological diagnoses were based on the test results alone ( $A D_{\text {Test }}$ vs. non$A D_{\text {Test }}$ ) irrespective of the comprehensive clinical diagnosis. In this context, a logistic regression model was implemented. This model included all CERAD subscales as independent variables and the $A D_{\text {PET }}$ vs. non$A D_{P E T}$ categorization as the dependent variable. All variables were entered in a single step and the respective non-significant predictor with the lowest significance was removed recursively until solely significant predictors were left in the model. Based on this final model, a cross tabulation was generated and the resulting dichotomous classification was matched against the FDG-PET/CT results.

Finally, a factor analysis was implemented to gain an impression of the convergent validity of the cognitive test results. All calculations were executed with SPSS 20.0.

\section{Results}

\section{Sample Characteristics}

Demographic and clinical characteristics are displayed in Table 1. The sample consisted of 127 patients, 33 of which were diagnosed with AD according to the results of the FDG-PET/CT. As displayed, the sample included diverse etiologies associated with cognitive decline, that were pooled into the two groups $A D$ and non-AD. One-way ANOVAs showed that these two groups did not differ significantly in age or sex, but that the AD-group had significantly fewer years of education and also a significantly lower mean score on the Mini Mental Status Examination (MMSE). The latter was a part of the CERAD test battery. 


\section{Congruency of neuropsychological and FDF-PET/CT diagnostic results: clinical decision model}

Estimates of sensitivity, specificity and overall accuracy for the clinical decision model are displayed in Table 2. Based on the comprehensive neuropsychological examination, 25 patients were diagnosed with AD, out of 33 patients who were categorized as such based on the FDG-PET/CT examination (sensitivity: 75.8\%). Out of the remaining 94 patients who were categorized as non-AD patients based on the FDG-PET/CT examination, 87 patients were categorized accordingly based on the neuropsychological examination (specificity: 92.6\%). Accuracy was estimated at $84.2 \%$.

Table 2 Congruency of the comprehensive neuropsychological and FDG-PET/CT diagnostic results

\begin{tabular}{|c|c|c|c|c|}
\hline & & \multicolumn{3}{|c|}{ FDG-PET/CT Diagnosis } \\
\hline & & $A D$ & Non & \\
\hline \multirow{6}{*}{$\begin{array}{l}\text { Neuro-psychological } \\
\text { Diagnosis }\end{array}$} & $A D$ & 25 & 7 & 32 \\
\hline & & \multicolumn{3}{|c|}{ Sensitivity: $75.8 \%$} \\
\hline & Non-AD & 8 & 87 & 95 \\
\hline & & & \multicolumn{2}{|c|}{ Specificity: 92.6\% } \\
\hline & & 33 & 94 & 127 \\
\hline & & & \multicolumn{2}{|c|}{ Balanced Accuracy: $84.2 \%$} \\
\hline
\end{tabular}

Note. Cross-tabulation with respective number of cases (total $n=127$ ). Displayed are results of the clinical decision approach in which the neuropsychological diagnosis incorporated anamnestic information obtained during the examination, behavioral observation during testing, as well as the evaluation of the test results

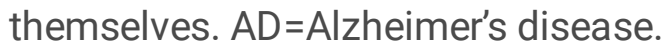

\section{Congruency of neuropsychological and FDG-PET/CT diagnostic results: logistic regression model}

Estimates of sensitivity, specificity and accuracy obtained for the logistic regression model that was based on the neuropsychological test results alone $\left(A D_{\text {Test }}\right.$ vs. non- $\left.A D_{\text {Test }}\right)$, are displayed in Table 2. In this model, respective recursive elimination of CERAD variables with the least significant contribution yielded four final variables with a significant contribution to the classification of patients in relation to the FDG-PET/CT diagnostics. Details of the recursive analytical steps are provided in Appendix 2. The four remaining variables included the total immediate recall performance on the episodic verbal memory task of the CERAD (wordlist total), as well as the delayed recall on the same task (wordlist delayed recall). Additionally, recall performance on the episodic non-verbal memory task remained as a significant predictor, specifically the difference 
between immediate and delayed recall performance on this task, i.e. memory trace decay (figure savings). Hence, as would be expected in context of differential diagnostics in $A D$, the indicated parameters were associated with the domain of episodic memory for new information. The final fourth variable reflected performance on a phonematic fluency task of spontaneous word generation (s-words). The latter reflects executive functioning, specifically divergent verbal problem solving and overall cognitive speed.

The resulting model with these four variables (wordlist total, wordlist recall, figure savings, s-words) explained $39.5 \%$ of variance according to Nagelkerke's $\mathrm{R}^{2}$, which reflects a moderate explanatory value. As depicted in Table 3, out of 32 patients categorized as AD patients based on the FDG-PET/CT, 18 were correctly identified based on this model. This reflected a low estimate of sensitivity of $56.3 \%$. On the other hand, estimates of specificity based on this model were excellent, as $93.3 \%$ of the non-AD patients categorized through FDGPET/CT were correctly identified based on the regression model. Accuracy reached a value of $74.8 \%$.

Table 3 Congruency of the neuropsychological diagnostics solely based on CERAD test results and the FDGPET/CT diagnostics

\begin{tabular}{|c|c|c|c|c|}
\hline & & \multicolumn{3}{|c|}{ FDG-PET/CT Diagnosis } \\
\hline & & $A D$ & Non & \\
\hline \multirow{6}{*}{$\begin{array}{l}\text { Neuro-psychological } \\
\text { Diagnosis }\end{array}$} & $A D$ & 18 & 6 & 24 \\
\hline & & Sen & & \\
\hline & Non-AD & 14 & 83 & 97 \\
\hline & & & \multicolumn{2}{|c|}{ Specificity: $93.3 \%$} \\
\hline & & 32 & 89 & 121 \\
\hline & & & \multicolumn{2}{|c|}{ Balanced Accuracy: $74.8 \%$} \\
\hline
\end{tabular}

Thus, the accuracy of the test-based logistic regression model was considerably lower than the accuracy of the clinical decision model. While estimates of specificity were comparable, the logistic regression model involved lower estimates of sensitivity (clinical approach: $75.8 \%$ versus test-based approach: $56.3 \%$ ), that yielded lower overall accuracy of the logistic regression model.

\section{Exploratory Factor Analysis}

In a post hoc analysis, cognitive test results of the CERAD were subjected to a factor analysis. The factor analysis was implemented to gain an impression of the convergent validity of the cognitive test results. Previous work on the German version of the CERAD has shown that the CERAD involves a three-factor structure and that results from verbal versus non-verbal episodic memory scales of the CERAD load on separate factors [17]. In the current work, the analysis of the underlying factor structure was conducted with a principal factor analysis using promax rotation. The structural matrix of the rotated solution is displayed in Table 4. All calculations were performed using z-scores of the respective CERAD scales. Five parameters were excluded from the analysis as their measure of sampling adequacy (MSA) was below .5, indicating that they were unsuitable for inclusion into the factor analysis (Boston Naming Test, Figure copy, Wordlist Trial 1, Trail- 
Making-Test A, Trail-Making-Test B/A). After exclusion of these five variables, the Bartlett-test was highly significant $(p<.001)$ and the Kaiser-Meyer-Olkin-criterion reached a value of .808, which can be considered good. Both results point to the suitability of the data for executing a factor analysis. Three factors with eigenvalues greater than 1 accounted for $71.22 \%$ of variance (Factor 1: $46.87 \%$, Factor 2: $12.97 \%$, Factor 3 : $11.38 \%$ ). Correlations of respective variables with factors suggested that the first factor reflected general verbal cognitive abilities, including verbal fluency and verbal short-term memory. The second factor reflected aspects of nonverbal memory and variables loading on the third factor reflected aspects of verbal memory. In sum, a three-factor structure emerged. Congruent with such a factor structure previously reported for the German version of the CERAD [17], verbal and non-verbal memory functions were distinguishable based on this factor solution.

Table 4 Structural matrix of the factor analysis

\begin{tabular}{|llll|}
\hline CERAD Variable & \multicolumn{3}{l|}{ Factor } \\
\hline Wordlist Total & 1 & 2 & 3 \\
\hline S-Words & .931 & .430 & .561 \\
\hline Animals & .710 & .277 & .036 \\
\hline Wordlist Trial 2 & .723 & .439 & -.006 \\
\hline Wordlist Trial 3 & .786 & .234 & .449 \\
\hline Figure Recall & .918 & .412 & .481 \\
\hline Figure Savings & .384 & .955 & .337 \\
\hline Wordlist Delayed Recall & .680 & .452 & .881 \\
\hline Wordlist Savings & .210 & .295 & .844 \\
\hline Wordlist Interference & .032 & .048 & .388 \\
\hline Wordlist Discrimination & .620 & .436 & .732 \\
\hline MMSE & .689 & .746 & .323 \\
\hline TMT-B & .667 & .640 & .257 \\
\hline
\end{tabular}

\section{Discussion/conclusion}

The purpose of the current study was to examine the congruency of neuropsychological differential diagnostics in AD on the one hand and FDG-PET/CT differential diagnostics on the other hand. In terms of the neuropsychological procedure, it was assumed that the comprehensive clinical procedure that relied on anamnestic information, behavioral monitoring during testing and the test results themselves, would be superior in accuracy, as compared to a data-driven procedure where the diagnostic decision was based solely 
on the results of the cognitive tests. The latter hypothesis implies that in routine clinical practice, the overall clinical impression of the neuropsychologist is of critical relevance for the differential diagnostic decision.

Results of the current study indicate that the clinical decision approach including a complete neuropsychological workup reached good accuracy (84.2\%) and yielded sufficient congruency with FDG$\mathrm{PET} / \mathrm{CT}$ diagnostics. This is in line with the extant literature concerning the use of the CERAD-NAB in context of comprehensive $A D$ diagnostics $[6,17,18]$. However, the test-based decision approach involved considerably lower accuracy $(74.8 \%)$, that was attributable to a low sensitivity estimate of only $56.3 \%$. The latter finding implies that about one third of patients with an AD diagnosis based on FDG-PET/CT diagnostics would remain undetected, if only the neuropsychological test results were considered. This implies that obtaining anamnestic information and behavioral monitoring during neuropsychological testing is highly relevant for differential diagnostic decisions in $A D$ patients.

It is notable that the current results emerged in a mixed, unselected sample including various etiologies that may account for cognitive deficits. The fact that such an unselected, mixed sample was included may be regarded as a strength of the current study, as it accurately reflects the actual circumstances in routine clinical practice. Yet, on the other hand one might argue that such uncontrolled circumstances may limit the validity of the current findings. In consideration of this issue, in a post hoc analysis, a factor analysis was implemented to examine the convergent validity of the neuropsychological test results with those from other studies. In previous work, factor analyses of the CERAD commonly revealed a three-factor solution $[17,19,20]$ and the same was the case for data of the current work. Albeit factor labeling may not be entirely consistent across studies, in particular the differentiation between verbal and non-verbal memory factors emerged as a common feature [17] and was also observable in the current work. These findings are generally compatible with the notion that the implementation of the neuropsychological procedure was methodologically sound in the current work. Moreover, it should be noted that in the logistic regression model of the test-based decision approach, two scales of verbal memory and one scale of non-verbal memory were among the four CERAD scales that predicted the classification of patients, relative to the FDG-PET/CT diagnostics. Hence, particularly memory parameters contributed to this decision model, which is in line with the notion that dementia and particularly $A D$ is characterized by memory deficits $[2,21]$.

In sum, these results imply that the methodological implementation of the neuropsychological tests was sound. Yet, test results by themselves yielded a considerably lower accuracy in predicting AD diagnoses based on FDG-PET/CT examinations than the clinical decision for which behavioral monitoring and anamnestic information were available to the clinician. In essence, these findings highlight the necessity of specialized and experienced staff involved in the neuropsychological diagnostics of AD. It is their expertise which allows the identification of behavioral abnormalities that - in conjunction with the neuropsychological test results - point towards a likely diagnosis of AD. Examples of such behavioral abnormalities that might show a certain specificity for mild $A D$ include depressive symptoms $[22,23]$ or anosognosia in the face of severe memory loss [24,25].

With regards to the sample included in the current work, it appears noteworthy that several demographic and clinical differences emerged between the AD and non-AD groups, as classified by the FDG-PET/CT (see Table 1). In particular, the AD groups scored significantly lower on the Mini Mental Status Examination 
(MMSE), that was a screening tool included in the CERAD test battery. Further, AD patients were characterized by significantly fewer years of education. In particular the latter has been described as a potential risk factor for $A D$ [26] and the fact that it emerged as a variable that differed across groups based on the FDG-PET/CT classification is in turn compatible with the notion that the applied FDG-PET/CT diagnostic procedure was methodologically sound.

Nevertheless, several limitations of the current study ought to be noted. First, even though FDG-PET/CT may be regarded as an accurate method to classify different forms of dementia, it may not provide an entirely error-free differentiation and does not represent a diagnostic gold-standard by itself. However, the purpose of the current work was to explore the congruency of different approaches to neuropsychological diagnostics in $A D$ with FDG-PET/CT and the research design of the current study met this issue. Secondly, it should be noted that even though it appears justified to conclude that a comprehensive neuropsychological approach including anamnestic information and behavioral monitoring during testing is superior to diagnostics based on test-results alone, it remains to be specified which of these areas of information is particularly relevant for the diagnostic decision. Future studies ought to address this issue in more detail in order to identify behavioral aspects such as awareness to deficits [25] that should be particularly attended to by clinicians as they might be specifically associated with $A D$.

\section{Declarations}

\section{Ethics approval and consent to participate}

This research was conducted ethically and in accordance with the World Medical Association Declaration of Helsinki. All participants provided written informed consent prior to study entry. The study was approved by the ethics committee of the University of Bamberg, Germany (reference number 2019-02/10).

\section{Consent for publication}

Not applicable.

\section{Availability of data and materials}

The data generated and analysed during the current study are available from the corresponding author on reasonable request.

\section{Funding Sources}

This study was supported by internal funding from the Klinikum Bayreuth $\mathrm{GmbH}$, Germany. The funding body did not influence study design, data collection, analysis, interpretation or writing of the manuscript.

\section{Author Contributions}

SH drafted and oversaw implementation of the study and drafted the manuscript, JK, KK, RM, JH and JKo supported recruitment and statistical analyses, PO oversaw implementation of the study, SF helped analyzing the data and drafting the manuscript, PK helped drafting and finalizing the manuscript. 
Acknowledgements

Not applicable.

\section{Supplementary Files}

This is a list of supplementary files associated with this preprint. Click to download.

- Appendix.docx 